\title{
Silicon Pore Optics development for ATHENA
}

Collon, Maximilien J.; Vacanti, Giuseppe; Guenther, Ramses; Yanson, Alex; Barriere, Nicolas; Landgraf, Boris; Vervest, Mark; Chatbi, Abdelhakim; Beijersbergen, Marco W.; Bavdaz, Marcos

Total number of authors:

22

Published in:

Proceedings of SPIE

Link to article, DOI:

$10.1117 / 12.2188988$

Publication date:

2015

Document Version

Publisher's PDF, also known as Version of record

Link back to DTU Orbit

Citation (APA):

Collon, M. J., Vacanti, G., Guenther, R., Yanson, A., Barriere, N., Landgraf, B., Vervest, M., Chatbi, A.,

Beijersbergen, M. W., Bavdaz, M., Wille, E., Haneveld, J., Koelewijn, A., Leenstra, A., Wijnperle, M., van Baren, C., Mueller, P., Krumrey, M., Burwitz, V., ... Christensen, F. E. (2015). Silicon Pore Optics development for ATHENA. In Proceedings of SPIE (Vol. 9603). [96030K ] SPIE - International Society for Optical Engineering. Proceedings of SPIE - The International Society for Optical Engineering https://doi.org/10.1117/12.2188988

\section{General rights}

Copyright and moral rights for the publications made accessible in the public portal are retained by the authors and/or other copyright owners and it is a condition of accessing publications that users recognise and abide by the legal requirements associated with these rights.

- Users may download and print one copy of any publication from the public portal for the purpose of private study or research.

- You may not further distribute the material or use it for any profit-making activity or commercial gain

- You may freely distribute the URL identifying the publication in the public portal 


\title{
Silicon Pore Optics development for ATHENA
}

\author{
Maximilien J. Collon ${ }^{1}$, Giuseppe Vacanti ${ }^{2}$, Ramses Günther ${ }^{2}$, Alex Yanson ${ }^{2}$, Nicolas Barrière $^{2}$, Boris Landgraf ${ }^{2}$, \\ Mark Vervest ${ }^{2}$, Abdelhakim Chatbi ${ }^{2}$, Marco W. Beijersbergen ${ }^{1}$, Marcos Bavdaz $^{3}$, Eric Wille ${ }^{3}$, Jeroen Haneveld ${ }^{4}$, \\ Arenda Koelewijn ${ }^{4}$, Anne Leenstra ${ }^{4}$, Maurice Wijnperle ${ }^{4}$, Coen van Baren ${ }^{5}$, Peter Müller ${ }^{6}$, Michael Krumrey ${ }^{6}$, \\ Vadim Burwitz ${ }^{7}$, Giovanni Pareschi ${ }^{8}$, Paolo Conconi $^{8}$, Finn E. Christensen ${ }^{9}$ \\ ${ }^{1}$ cosine Research B.V, J.H. Oortweg 19, NL-2333 CH Leiden \\ 2 cosine Science \& Computing B.V, J.H. Oortweg 19, NL-2333 CH Leiden \\ ${ }^{3}$ European Space Agency, ESTEC, Keplerlaan 1, PO Box 299, 2200 AG Noordwijk, The Netherlands \\ ${ }^{4}$ Micronit Microfluidics B.V., Colosseum 15, 7521 PV Enschede, The Netherlands \\ ${ }^{5}$ SRON, Sorbonnelaan 2, 3584 CA Utrecht, The Netherlands \\ ${ }^{6}$ Physikalisch-Technische Bundesanstalt (PTB), Abbestr. 2-12, 10587 Berlin, Germany \\ ${ }^{7}$ MPI f. extraterrestrische Physik, Giessenbachstrasse 1, 85748 Garching, Germany \\ ${ }^{8}$ INAF Osservatorio Astronomico di Brera, Via E. Bianchi 46 I- 23807, Merate (Lc), Italy \\ ${ }^{9}$ DTU Space, Technical University of Denmark, Building 327, DK - 2800 Kgs. Lyngby, Denmark
}

\begin{abstract}
The ATHENA mission, a European large (L) class X-ray observatory to be launched in 2028, will essentially consist of an X-ray lens and two focal plane instruments. The lens, based on a Wolter-I type double reflection grazing incidence angle design, will be very large $(\sim 3 \mathrm{~m}$ in diameter) to meet the science requirements of large effective area $\left(1-2 \mathrm{~m}^{2}\right.$ at a few $\left.\mathrm{keV}\right)$ at a focal length of $12 \mathrm{~m}$. To meet the high angular resolution $(5 \mathrm{arc}$ seconds) requirement the X-ray lens will also need to be very accurate. Silicon Pore Optics (SPO) technology has been invented to enable building such a lens and thus enabling the ATHENA mission. We will report in this paper on the latest status of the development, including details of X-ray test campaigns.
\end{abstract}

Keywords: X-ray optics, X-ray astronomy, silicon, wafer, stack, pore optics, X-ray telescopes, ATHENA, SPO

\section{INTRODUCTION}

Silicon Pore Optics (SPO) is a high-energy optics technology developed with funding of the European Space Agency (ESA) by a consortium of industrial and institutional partners. SPO were invented in $2002[1,2]$ to enable future x-ray observatories that would succeed the then newly launched CHANDRA [3] and XMMNewton [4] telescopes. The technology development progressed over the years from a proof of concept stage to a very mature x-ray optic that now forms the basis of the Advanced Telescope for High Energy Astrophysics (ATHENA), the second (L2) large class mission with the ESA Cosmic Vision Program [5], selected in June 2014.

This selection also boosted the efforts for the technology development, which after years of changing mission scenarios, does now focus on pushing the optics to meet the ATHENA angular resolution and effective area requirements.

\section{SILICON PORE OPTICS}

$\mathrm{X}$-ray optics for space applications, operating at from a hew hundreds of $\mathrm{eV}$ up to tens of $\mathrm{keV}$, are in general based on grazing incidence mirrors that need to be fabricated and mounted. The mirrors need to have a roughness of better than a few Ångstrom to efficiently reflect the x-rays and must have an accurate figure and be co-aligned to achieve high angular resolution. The effective area requirement results in optical designs that maximize the amount of mirrors whilst the mass limitations imposed by the launcher demand very thin mirrors. A number of technologies, such as slumped glass [6-8], silicon foils [9], glass or silicon micro-pores [10-15] are being developed to make the required lightweight X-ray optics.

Optics for EUV, X-Ray, and Gamma-Ray Astronomy VII, edited by Stephen L. O'Dell, Giovanni Pareschi, Proc. of SPIE Vol. 9603, 96030K · C 2015 SPIE · CCC code: 0277-786X/15/\$18 · doi: 10.1117/12.2188988 
SPO relies on products and processes of the semiconductor industry, which has invested heavily into making silicon wafers, normally used to produce electronics and memory chips as used for example in smart phones. Semiconductor fabs cut the wafers from single crystalline silicon ingots, grown in large factories from polycrystalline silicon, and then grind them several times to improve the thickness uniformity (total thickness variation - TTV). After rounding the edges and an etching step to remove surface layer damages one loads up to 15 wafers at the same time into a double side polishing (DSP) machine, which reduces the roughness of the wafer down to better than $0.1 \mathrm{~nm}$. The DSP machines are able to produce large batches of wafers with the same absolute thickness, with a standard deviation of less than $0.3 \mu \mathrm{m}$. Within each wafer the TTV is less than $300 \mathrm{~nm}$ (peak-to-valley) and typically $30 \mathrm{~nm}$ on the central $90 \%$ of the area. Each wafer has a unique ID and one can obtain also data sets per wafer with thickness maps. This results in almost perfect, single crystalline, silicon disks, which are thin, smooth, plan parallel and very clean - the perfect (x-ray) mirror (see Figure 1).

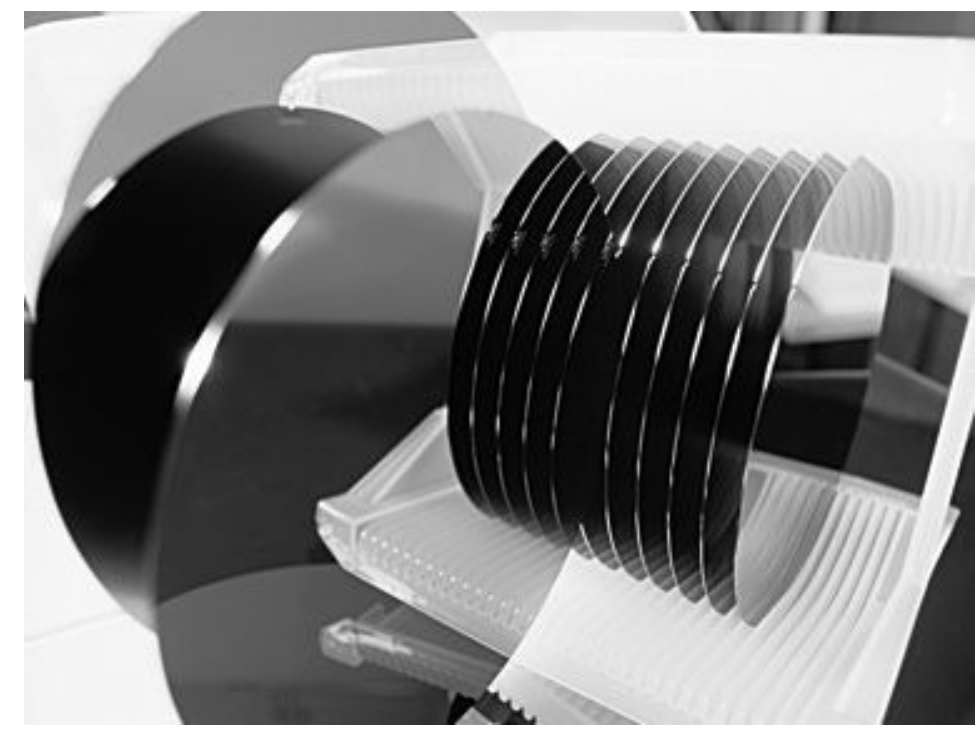

Figure 1 A box with $300 \mathrm{~mm}$ diameter double-sided polished silicon wafers

Silicon wafers need to be further processed into plates, for which we rely on many existing suppliers that deliver mass production equipment using standardized processes to structure, etch, cut, coat and transport silicon wafers. The plates, being diced in rectangular shape from the wafer, have two main functional parts: a membrane, acting as the mirror, and ribs to interconnect multiple plates. In addition one has the possibility to add a wedge to the plate, depending on the type of optics one wants to later build. For example, to make Wolter-I type [16] optics, a wedge must be added to each pair of plates to make the angle at which on axis x-rays meet the primary mirror at each radius comply with the Wolter-I configuration and its approximations.

The plates are then elastically bent and stacked on top of each other by bonding them together. This stacking process is the core of the SPO technology. In this step the plate is prepared for bonding, is bent to the desired figure and is then bonded on top of other plates. The important point is that the plates are bonded directly, without any glue, by means of direct hydrophilic bonding of activated surfaces. This technique originally stems from optical bonding techniques to join lenses or prisms together [17]. The silicon plates are first being cleaned to remove any residual particulate contamination, preferable down to a size of $100 \mathrm{~nm}$. Then the bonding surfaces need to be activated. This is done either by wet activation using a dip of the ribbed plate in a set of chemicals or by using ambient plasma activation. 


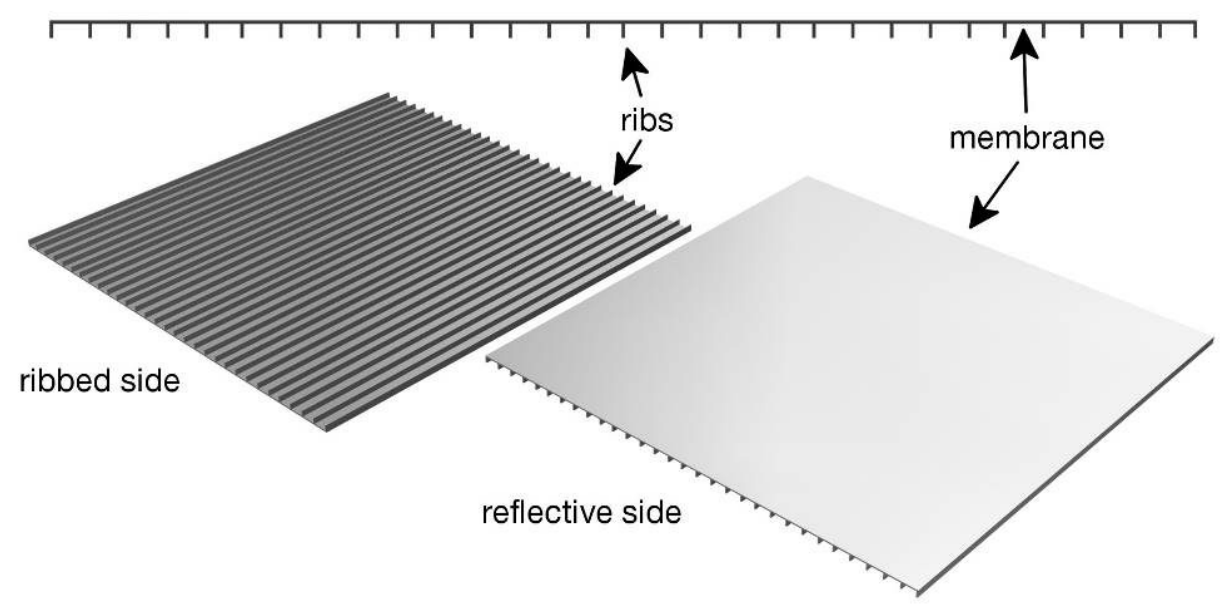

Figure 2 A diced plate has a ribbed side and a reflective side. The ribs are formed by a grooving process using a dicing machine. The membrane is left at the bottom of the groove.

Both methods result in a hydrophilic silicon plate and a rib surface that is covered in hydroxyl groups. When two of such plates are brought into close contact at room temperature a natural water interlayer joins the hydroxyl groups, a so-called pre-bond, by means of Van-der-Waals forces. During annealing at elevated temperatures $\left(>110^{\circ} \mathrm{C}\right)$ a polymerisation reaction takes place that drives the water out and produces higher strength covalent bonds.

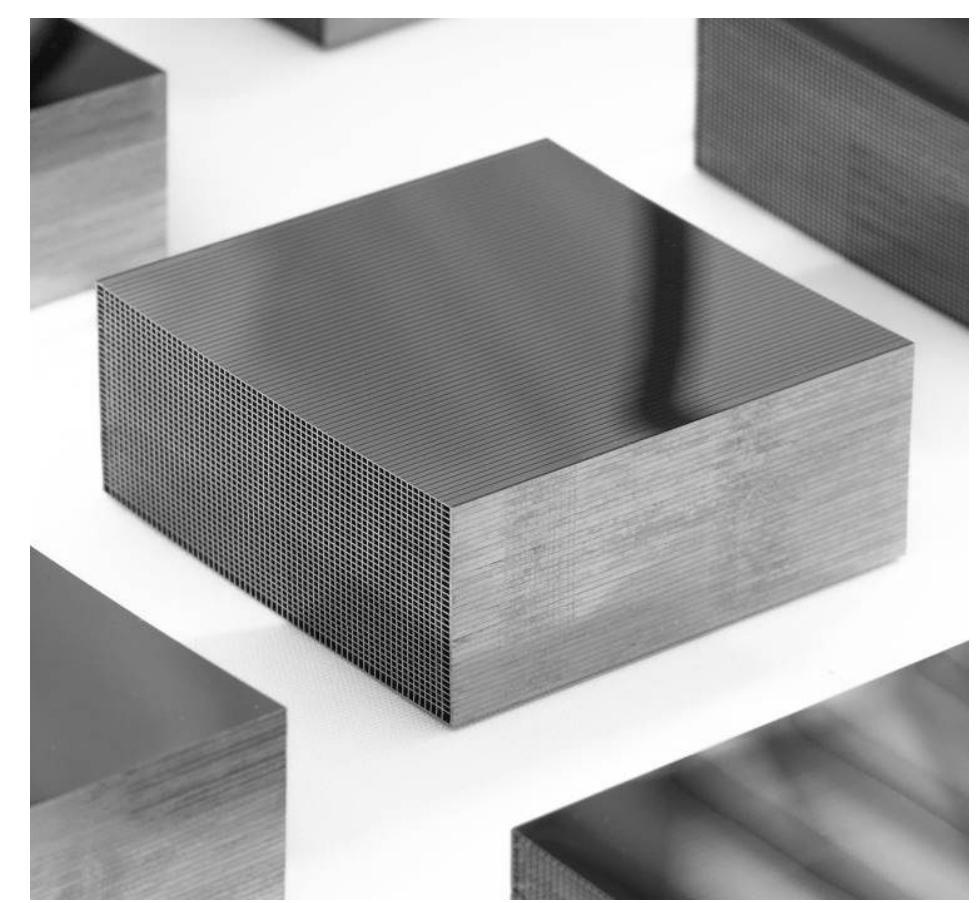

Figure 3 A stack of 35 silicon plates including coating. That particular stack has dimensions of $66 \times 66$ $\mathrm{mm}^{2}$, an outer radius of curvature of $0.74 \mathrm{~m}$ and consists of $\sim 2100$ pores with a rib and membrane width of $0.17 \mathrm{~mm}$, resulting in an open area ratio of more than $60 \%$. 
The cleaned and activated silicon plates are loaded into a fully automated stacking robot, which we developed especially for SPO production. The stacking robot operates in a class 100 cleanroom and fits onto an optical table. The plates are being inspected for cleanliness and are then brought by a robotic arm to a pre-bending station, the so-called die. This is a convex surface that brings the plate into the approximate shape required for the stack, with the ribs hanging downwards.

The robot then aligns a concave silicon mandrel to the plate on the die. The mandrels are responsible, contrary to other x-ray optics, only for the figure of a stack, the roughness of the plates is however not influenced. A mandrel has either a cylindrical, conical or even a secondary curvature figure that the stack will copy. Mandrels are marginally larger in size than the plates and can be cleaned and recycled without refurbishment. A mandrel is produced by coarse grinding and ion beam figuring of a silicon block, which can be done by a number of companies and which is a process routinely used to produce synchrotron optics with a residual figure error of 0.1 $\mathrm{nm}$. Note that the silicon mandrel material matches the coefficient of thermal expansion (CTE) of the stack.

To start a stack, the mandrel is equipped with a so-called base plate. The robot the presses the plate hanging on the die onto the base-plate and, after release of the plate from the die, a small stack of two plates is formed. The figure of the stack is measured using interferometric metrology. The robot then picks the next plate, pre-bends it using the die and repeats the bonding process. This cycle goes on until a stack of 20-45 plate height is reached, depending on the optics design. Note that the mandrel only determines the outer most radius of the stack.

The stack is then removed from the mandrel and forms a free-standing, light-weight, stiff, silicon block, with a large open area ratio (see Figure 3).

The stack quality mainly defines the angular resolution of the X-ray optic. To form an optic, we align and mount two stacks behind each other and fix their relative position using brackets, which are CTE matched to the silicon. This step is done at an x-ray facility to test the optics at the wavelength it later needs to operate in. The result is a mirror module, essentially an x-ray lens (see Figure 4).

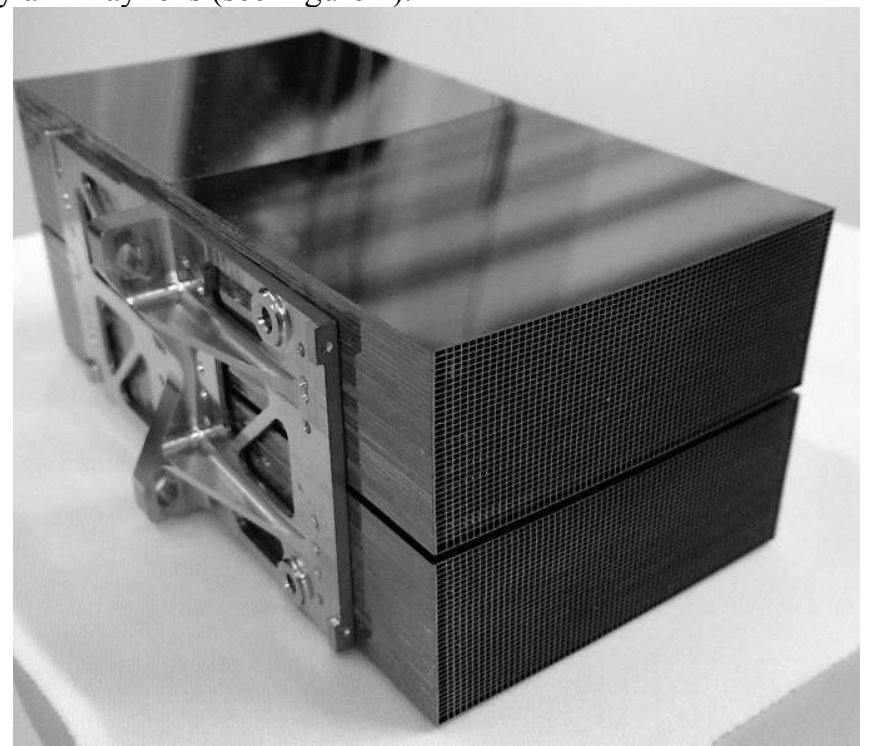

Figure 4 A complete SPO mirror module, consisting of $2 \times 2$ stacks of 35 plates, held together by high precision Invar brackets, which form the interface to the optical bench.

\section{TECHNOLOGY DEVELOPMENT}

The SPO development in the past focused on demonstrating in a holistic approach the entire production chain, from wafer up to optical bench level, at one focal length and radius, on which we reported extensively in [1833]. This included a complete set of environmental tests on mirror module level, resulting in a technology readiness level (TRL) of $\sim 5$ at the current stage. Up until the ATHENA mission selection only one stacking robot was available, which was frequently being modified and upgraded to follow the mission evolution, from producing $\mathrm{f}=50 \mathrm{~m} / \mathrm{r}=2000 \mathrm{~mm}$ stacks to middle radii $\mathrm{f}=20 \mathrm{~m} / \mathrm{r}=740 \mathrm{~mm}$ stacks. Recently the efforts were shifted to improving the angular resolution and to becoming able to build mirror modules at three representative radii of 
the ATHENA optics [35], namely the inner most $(\mathrm{r}=250 \mathrm{~mm})$, the middle $(\mathrm{r}=740 \mathrm{~mm})$ and the outer most radius $(\mathrm{r}=1500 \mathrm{~mm})$. As ATHENA will operate close to $\mathrm{f}=12 \mathrm{~m}$, the ESA technology development initiated the setup of additional stacking robots.

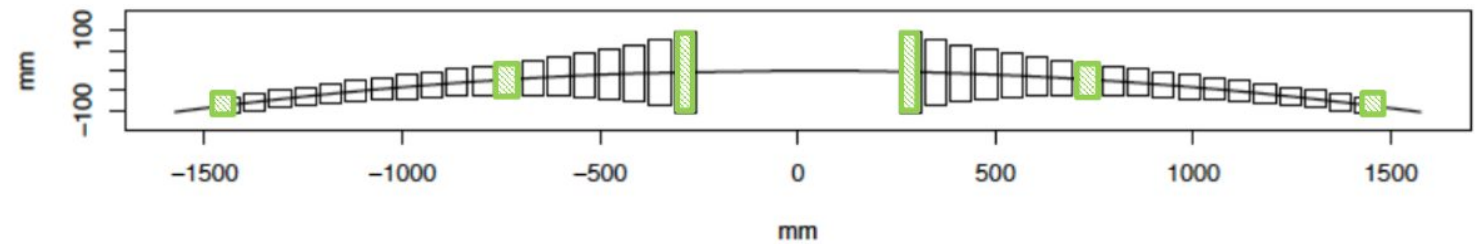

Figure 5 Cross section of the current design of the optical bench of ATHENA. The green boxes denote the three radial positions that the technology development focuses on.

ESA structures technology development in separate projects that each have their specific goals. The inner radii activity shall produce a robot that is capable of producing a first stack of plates with a length of $110 \mathrm{~mm}$ and a bending radius of $250 \mathrm{~mm}$. For this activity new plate types, wedging strategies and ribbing processes need to be developed. All of those developments benefit also all other radii, as for example the larger rib spacing and thinner membranes increases the overall effective area. The new, uniform, wedge distribution over both primary and secondary stack simplifies plate production and handling, as only one plate type is required in the future. At the same time we will optimise the bond strength, which profits stack integrity and ruggedisation efforts. The outer radii activity needs to deal with rather short plates, which pushes the cleanliness of the plate, which also benefits angular resolution, effective area and stack robustness. The efforts for inner and outer radii are being discussed in more detail in a separate paper by E. Wille in the same proceedings.

The majority of the efforts however currently are put into middle radii stacks, where the goal is to push the angular resolution of SPO, first at $\mathrm{f}=20 \mathrm{~m}$ and then at $\mathrm{f}=12 \mathrm{~m}$. We started by reviewing and improving the entire production chain. Initial mirror modules had an angular resolution half-energy width (HEW) of about 20" and we identified problems in the mandrel fabrication, the wedge uniformity, the plate cleanliness and the stacking process [32].

We first addressed the mandrel fabrication. The mandrel figure had an erroneous convex figure, which was corrected, resulting in a double reflection X-ray measurement of 6.3 " HEW on $10 \%$ of the area of a small mirror module (see Figure 6) at the PANTER test facility of the Max Planck Institut für extraterrestrische Physik in Munich [35].

As can be also seen from Figure 6 the other $90 \%$ of the area was disturbed by particulate contamination, which was traced to be the result of the final ion beam figuring process on mandrel fabrication. We introduced a super polishing step and the result can be seen in Figure 7, comparing one plate stacked on a mandrel before and after super polishing.

The vastly improved mandrel cleanliness however introduced a new issue: The super clean silicon mandrels started to nicely bond to the silicon stacks, rendering them difficult to release after production. We are currently developing passivation methods that on the one hand keep the mandrel clean and on the other hand prevent bonding of the mandrel. At the time of writing this paper this process is not finished, nevertheless, we were able to produce another test mirror module consisting of 20 plates, where we measured at $2.8 \mathrm{keV}$ in double reflection at the X-ray pencil beam facility (XPBF) in the PTB laboratory at the BESSY II synchrotron radiation facility in Berlin [36] on one third of the mirror module area HEW of 10.5" (see Figure 8). The other $70 \%$ of the area are disturbed by the afore mentioned not yet perfected mandrel passivation process. 


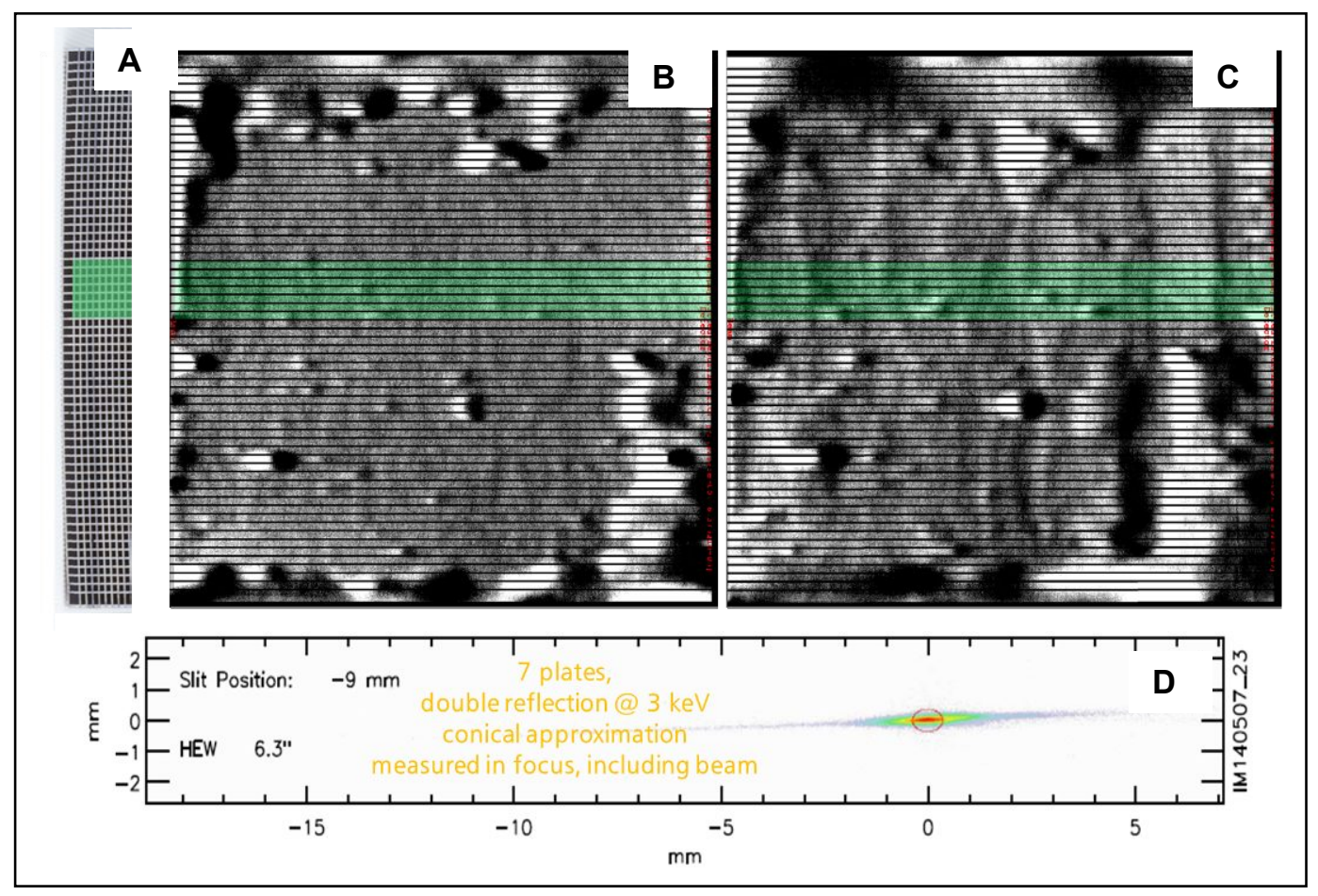

Figure 6 Measurement results of a small ( 7 plate) $\mathrm{r}=0.74 \mathrm{~m}$ test mirror module, fabricated using conical mandrels with a corrected figure (see text). (A) shows the mirror module from the front, (B) shows the production surface deviation metrology data of the first plate of the primary stack, (C) the same view as (B) of the secondary stack. (D) shows the resulting PSF measured at PANTER. The consecutive area marked in green yields in double reflection a HEW of 6.3". The grey areas are affected by ion beam figuring deposits, which have been removed since (see Figure 7).

We also measured a vastly reduced stack up error, demonstrating the improvements in plate quality and cleanliness (see Figure 9), where within the measurement accuracy we were no able to detect a significant deterioration of the stack quality.
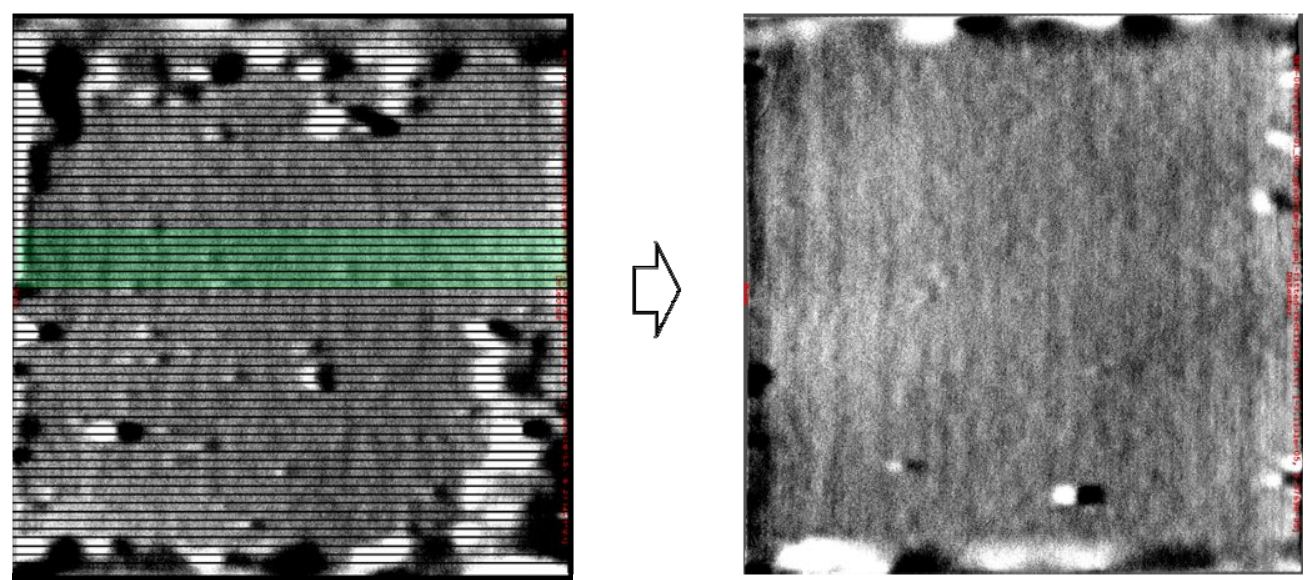

Figure 7 The left side shows the same first plate of the primary stack as in Figure 6, the right shows the first plate of a new stack made on the mandrel after superpolishing. Almost all of the ion beam figuring residue has been removed and the bonded plate shows only a few sub $100 \mathrm{~nm}$ sized particles. 


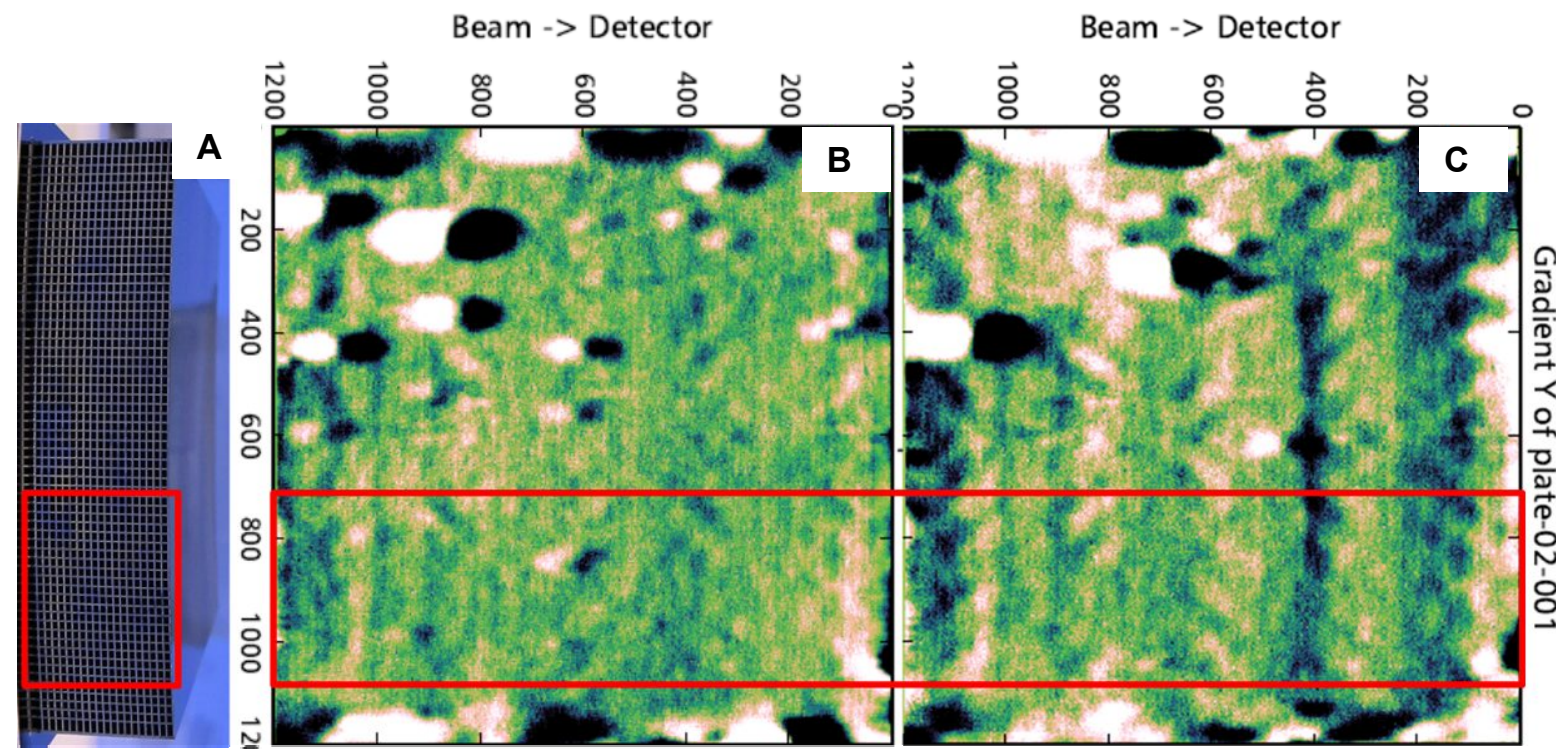

Figure 8 Measurement results of a 20 plate $r=0.74 \mathrm{~m}$ test mirror module, fabricated using super polished and passivated conical mandrels (see text). (A) shows the mirror module from the front, (B) the production surface deviation metrology data of the second plate of the primary stack, (C) the same view as (B) of the secondary stack. The consecutive area marked in red $(30 \%$ of the total area) yields in double reflection, measured at the XPBF in the PTB laboratory at BESSY, at $20 \mathrm{~m}$ a PSF with a HEW of 10.5". The other area is affected by the imperfect passivation process.

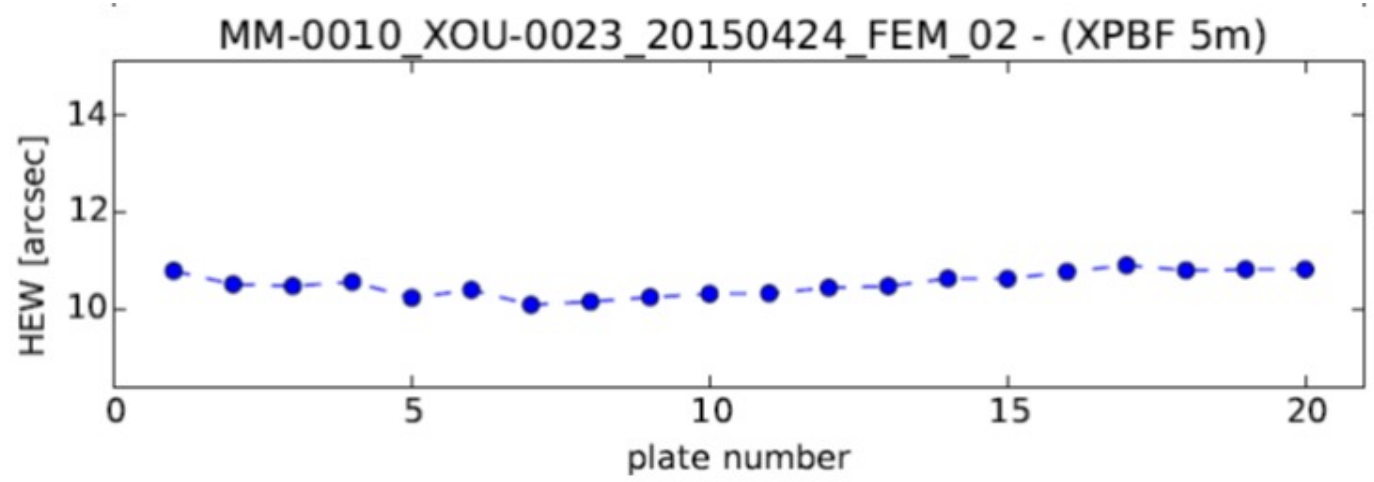

Figure 9 Cumulative HEW as a function of stack height of the mirror module shown in Figure 8, measured at $2.8 \mathrm{kEV}$ in the focus at $20 \mathrm{~m}$. The mirror module quality remains nearly constant with the measurement accuracy of 1 ".

Recently we have fabricated another test mirror module consisting of 20 plates with an improved passivation process, of which we show first test results in Figure 10. We have been able to increase the clean and well bonded area to $70 \%$ of mirror module surface (compare to Figure 8), of which the PSF at $20 \mathrm{~m}$ has been measured at $2.8 \mathrm{keV}$ in double reflection at the XPBF in the PTB laboratory at BESSY II to be 11.9" (see Figure 10). Again, we found the cumulative stack up error to remain constant as a function of stack height (not shown). The area outside the red box in Figure 10 is mainly affected by imperfections in the die geometry, which will be improved in the near future. 

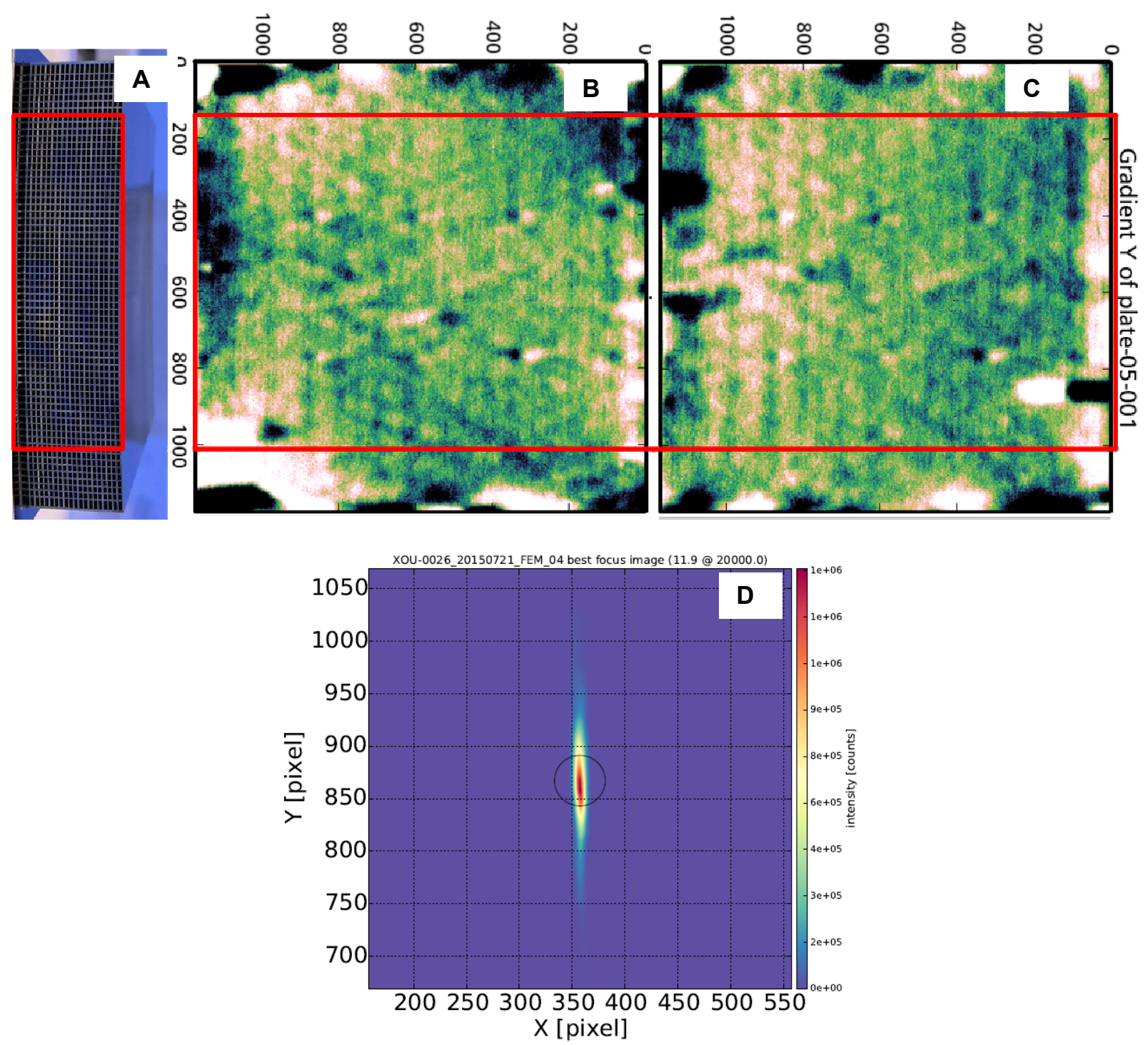

Figure 10 Measurement results of a 20 plate $r=0.74 \mathrm{~m}$ test mirror module, fabricated using an improved mandrel passivation process (see text). (A) shows the mirror module from the front, (B) the production surface deviation metrology data of the $5^{\text {th }}$ plate of the primary stack, (C) the same view as (B) of the secondary stack. The consecutive area marked in red $(70 \%$ of the total area) yields in double reflection, measured at $2.8 \mathrm{keV}$ at the XPBF in the PTB laboratory at BESSY, at $20 \mathrm{~m}$ a PSF with a HEW of 11.9" (D). Note that the data includes the probe pencil beam of 2".

Further analysis of the data showed that the stack quality is now mainly driven by the interface of the mandrel to the $1^{\text {st }}$ plate and as a consequence we are now currently optimizing the geometry of the die and the way we deposit the first plate. This is an iterative improvement process, depicted in Figure 11, for which we also upgrade the surface metrology systems that measure each plate during stacking to predict the $\mathrm{x}$-ray performance and to feed back into the production (see the proceedings of M. Bavdaz in the same conference). From these improvements we expect to be able to fabricate 0.74 m mirror modules with an angular resolution of better than 10 " in coming months. We will then proceed to push the angular resolution at $20 \mathrm{~m}$ to beyond 5" and will then modify the middle radius stacking robot to build stacks for a $12 \mathrm{~m}$ focal length. The modifications concern mainly the length of the plate, which in turn requires shorter mandrels and a shorter die. These process changes will be implemented in 2016. 


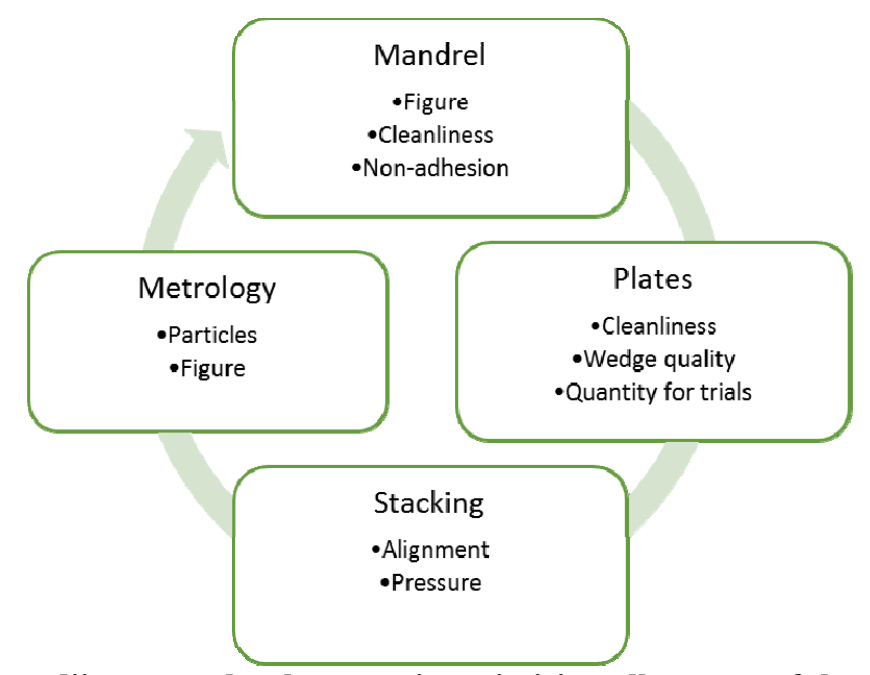

Figure 11 The middle radii process development is optimising all aspects of the stacking process in an iterative way. We work on all aspects of the stack production chain. The progress is regularly checked by fabrication of test stacks, which are also subject to $x$-ray testing.

To further facilitate the stacking process development we require a large quantity of plates to be manufactured, at a lower cost. To this end new dicing and ribbing machines have recently been procured (see Figure 12), which are capable of ribbing entire $300 \mathrm{~mm}$ wafers in one go. We have successfully produced first test samples (see Figure 13) and are currently commissioning the system.

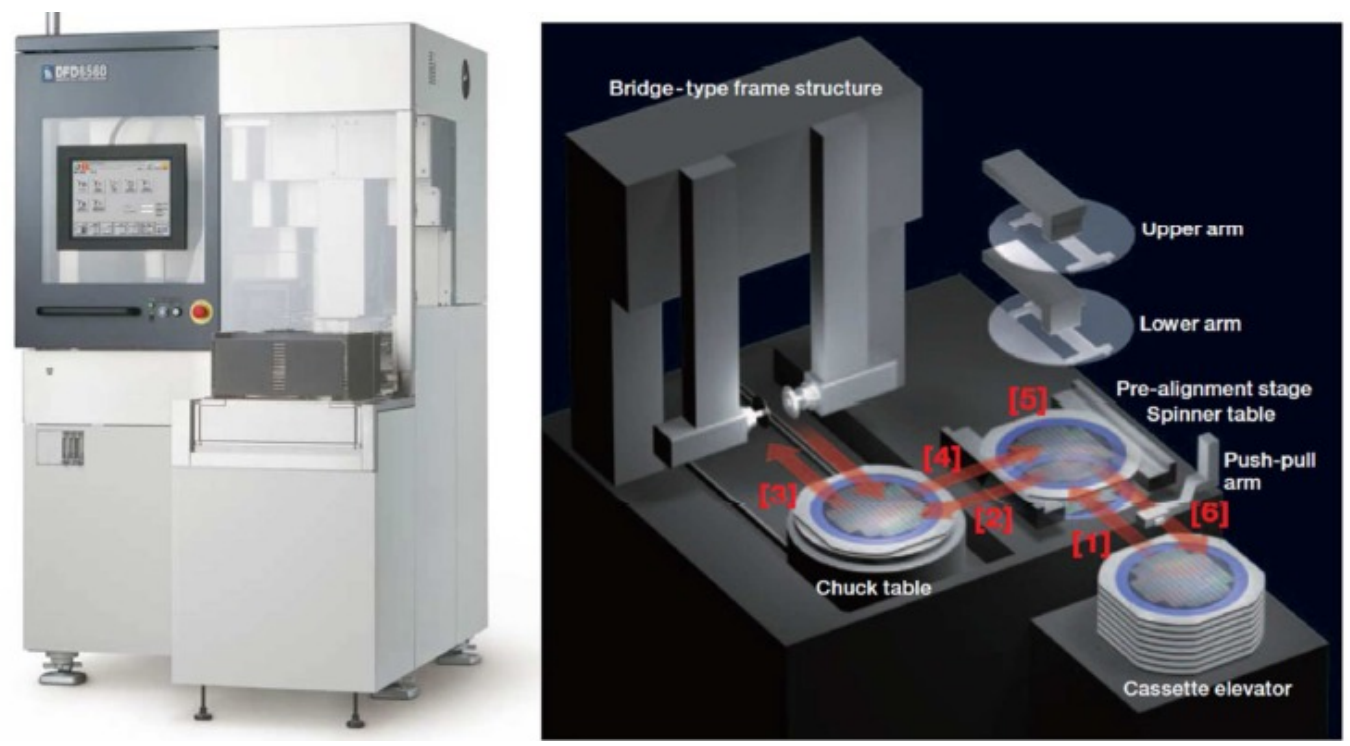

Figure 12 New fully automated dicing machine that has been procured to process 12" wafers in one go.

In parallel we invest into new wet benches, upgrades of die and optimise the stacking robot to reduce the stacking time, which in turn allows performing faster process optimisation. 

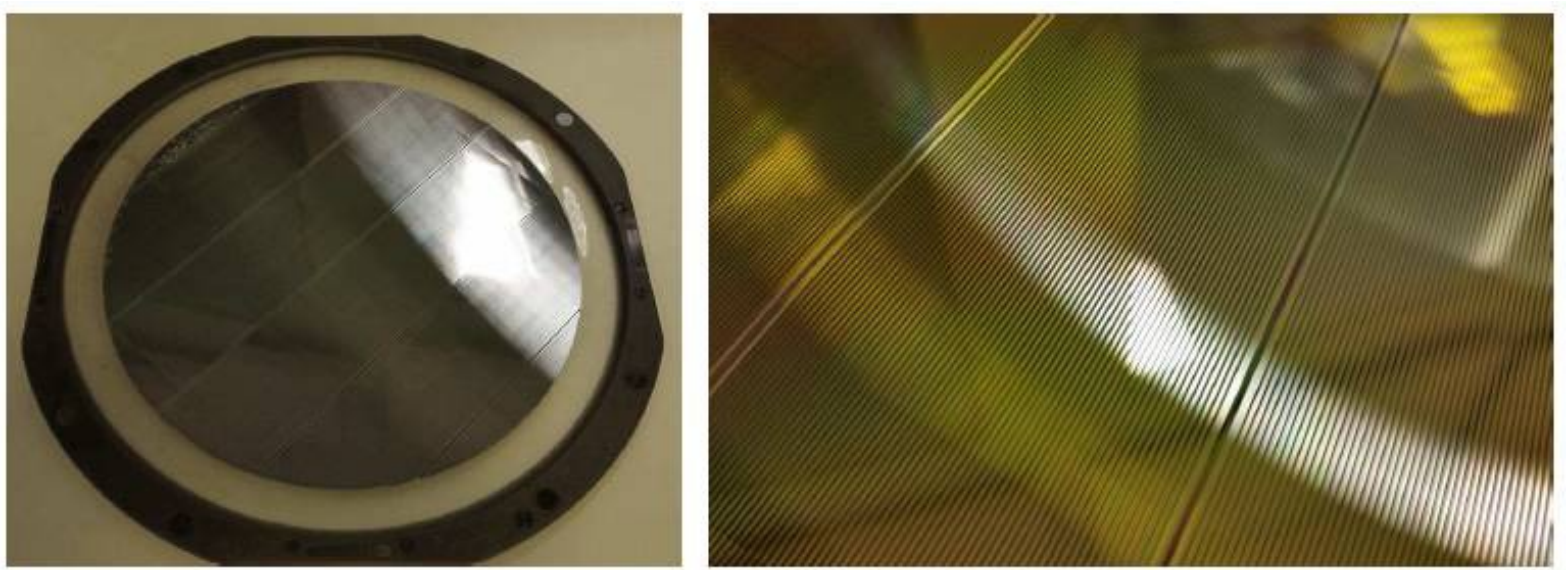

Figure 13 First sample of a 12" wafer that was diced and ribbed in one go.

\section{CONCLUSIONS}

Since the selection of ATHENA as the second large ESA mission in the Cosmic Vision program in June 2014 we have been increasing the development efforts to advance the Silicon Pore Optics developments at all fronts. The development focuses on improving the angular resolution at middle radii of $0.74 \mathrm{~m}$ and a focal length of 20 and will in 2016 change to $12 \mathrm{~m}$ focal length. X-ray tests on 20 plate pair mirror modules show that we currently reach an angular resolution of 10.5 " on $30 \%$ of the area and 11.9 " on $70 \%$ of the area, with a constant plate quality as a function of stack height. $10 \%$ of the optic are as good as 6.3 ". The near future development focuses on improving the interface of mandrel and $1^{\text {st }}$ plate. In parallel we stabilise and increase the production capacity by increasing the plate production rate and by reducing the stacking time.

The development of inner $(\mathrm{r}=0.25 \mathrm{~m})$ and outer $(\mathrm{r}=1.5 \mathrm{~m})$ stacking robots has commenced and first samples will become available in 2016. All of these efforts will allow making SPO mirror modules that enable the adoption of the ATHENA by 2020.

\section{REFERENCES}

[1] Beijersbergen, M. et al., "Silicon pore optics: novel lightweight high-resolution X-ray optics developed for XEUS", Proc. SPIE 5488, 868-874 (2004).

[2] Bavdaz, M. et al., "Progress at ESA on high-energy optics technologies", Proc. SPIE 5168, 136-147 (2004).

[3] O’Dell, S. L. et al., "Advanced X-ray astrophysics facility (AXAF): Calibration overview”, Proc. SPIE, 3444 (1998).

[4] Jansen, F. et al., "XMM-Newton observatory", Astron. Astrophys. 365, L1-L6 (2001).

[5] K. Nandra. Athena: Exploring the Hot and Energetic Universe. In Proc. American Astronomical Society HEAD meeting (\#14), (2014).

[6] Zhang, W. et al., "Development of Lightweight X-Ray Mirrors for the Constellation-X Mission", Proc. SPIE 6688, 668811 (2007).

[7] Ghigo, M. et al., " The manufacturing of the XEUS x-ray glass segmented mirrors: status of the investigation and last results ", Proc. SPIE 5168, 180 (2004).

[8] Friedrich, P. et al., "Manufacturing of Wolter-I mirror segments with slumped glass", Proc. SPIE 6266, 62661G (2006).

[9] Hayashi, T. et al, "Improvement of the angular resolution of a thin-foil-nested x-ray telescope", Proc. SPIE 7448, 74480A (2009).

[10] Wilkins, S. W. et al., "On the concentration, focusing, and collimation of x-rays and neutrons using microchannel plates and configurations of holes", Review of Scientific Instruments 60(6), 1026-1036 (1989).

[11] Beijersbergen, M. W. et al., "Microchannel-plate-based x-ray optics", Proc. SPIE 3765, p. 452-458 (1999). 
[12] Collon, M. J. et al., "X-ray imaging glass micro-pore optics”, Proc. SPIE 6688, pp. 668812 (2007).

[13] Wallace, K. et al., "Breadboard micro-pore optic development for x-ray imaging”, Proc. SPIE 6688, pp. 66881C (2007).

[14] Fraser, G, et al., "The mercury imaging X-ray spectrometer (MIXS) on Bepicolombo", Planetary and Space Science 58(1-2), 79 - 95 (2010).

[15] Ezoe, Y. et al., "Micropore x-ray optics using anisotropic wet etching of (110) silicon wafers", Applied Optics 45, $8932-8938$ (2006).

[16] Wolter, H., "Spiegelsysteme streifenden Einfalls als abbildende Optiken für Röntgenstrahlen", Annalen der Physik 445, 94-114 (1952).

[17] G. Wallis and D. I. Pomerantz, Field Assisted Glass-Metal Sealing, Journal of Applied Physics 40(10), 3946 (1969).

[18] Kraft, S. et al., "Development of modular high-performance pore optics for the XEUS x-ray telescope", Proc. SPIE 5900, 297-308 (2005).

[19] Günther, R. et al., "Production of silicon pore optics", Proc. SPIE 6266, 626619 (2006).

[20] Collon, M. J. et al., "Performance characterization of silicon pore optics", Proc. SPIE 6266, 62661T (2006).

[21] D. H. Lumb, F.E. Christensen, C.P. Jensen and M. Krumrey, "Influence of a carbon over-coat on the X-ray reflectance of XEUS mirrors", Opt. Commun. 279, 101 - 105 (2007).

[22] Olde Riekerink, M. B. et al. "Production of silicon mirror plates", Proc. SPIE 7437, 74370U (2009)

[23] Jensen, C. P. et al., "Coating of silicon pore optics”, Proc. SPIE 7437, 7437 (2009).

[24] Wallace, K. et al, "Silicon pore optics development", Proc. SPIE 7437, 7437 (2009).

[25] Kampf, D. et al., "Optical bench elements (petals) for IXO”, Proc. SPIE, 7437 (2009).

[26] Collon, M. J., et al., "Stacking of silicon pore optics for IXO", Proc. SPIE, 7437 (2009).

[27] Vacanti, G, et al., "Silicon pore optics for astrophysical missions", Proc. SPIE 7732, 773240 (2010).

[28] Ackerman, M, et al., "Compatibility of silicon pore optics with launchers and operation conditions", Proc. SPIE 8147, 81470D (2011).

[29] Bavdaz, M., "ESA optics technology preparation for IXO”, Proc. SPIE 7732, 77321E (2010).

[30] Wille, E., et al., "Mass Production of Silicon Pore Optics for IXO and ATHENA", Proc. SPIE 8147, 81470D (2011).

[31] COLLON 2014

[32] BAVDAZ 2014

[33] WILLE 2014

[34] R. Willingale, G. Pareschi, F. Christensen, J-W. den Herder, "The Optical Design of the Athena+ Mirror", An ATHENA+ supporting paper, $<\mathrm{http}: / /$ www.the-athena-X-ray-observatory.eu/>

[35] Freyberg, M. et al., "Potential of the PANTER x-ray test facility for calibration of instrumentation for XEUS", Proc. SPIE 6266, 62663H (2006).

[36] Krumrey, M., et al, "X-ray pencil beam facility for optics characterization”, Proc. SPIE 7732, 773240 (2010). 